\title{
Interfacial waves of finite amplitude
}

\section{Les ondes interfaciales d'amplitude finie}

\author{
J. N. HUNT \\ DEPARTMENT OF MATHEMATICS, IMPERIAI, COLLEGE, JONDON \\ I. - PROGRESSIVE WAVES \\ I. - ONDES PROGRESSIVES.
}

\begin{abstract}
Levi-Civita's method for progressive waves of finite amplitude is extended to the case of waves at a fluid interface, the wave profile and velocity being found to the third order. The principal effect of the upper fluid is to reduce the velocity of propagation and the amplitude of the higher harmonics in the wave profile.
\end{abstract}

\begin{abstract}
La méthode de Levi-Civita pour les ondes progressives d'amplitude finie est étendue au cas des ondes présentes à l'interface séparant deux fluides, l'expression du profil et de la vilesse de l'onde étant du troisième ordre. Le rôle principal da fluide supérieur consiste à diminuer la vitesse de propagation et l'amplitude des harmoniques supérieurs dans le profil d'onde.
\end{abstract}

\section{Introduction.}

Levi-Civita's proof [1], of the existence of progressive gravity waves of finite amplitude is extended to the case of waves at the interface between two semi-infinite fluids. The equation to the wave profile and the velocity of propagation are found to the third order in the ratio of wave amplitude to wavelength. The solution for waves at the interface between fluids of finite depth is also given. The amplitudes of the second and third harmonics are reduced by the presence of the upper fluid so that the wave profile is more sinusoidal than that of the progressive wave at a free surface. The velocity of propagation is also reduced.

Some modification of the convergence proof is found desirable and may be used to estimate the range of wave amplitudes whose existence has been rigorously established, as indicated elsewhere for waves at a free surface, Hunt [2]. It is shown how an alteration in the transformations used by Levi-Civita leads to an estimate of the amplitude of the highest wave, although the result is less accurate than that given by Yamada's method [3].

\section{Introduction.}

La preuve, apportée par Levi-Civita [1], de l'existence d'ondes de gravité d'amplitude finie, est étendue au cas des ondes à l'interface séparant deux fluides semi-infinis. L'équation du profil de l'onde et de la vitesse de propagation est du troisième ordre dans le rapport de l'amplitude à la longueur d'onde. La solution est également indiquée pour les ondes à l'interface de deux fluides de profondeur finie. Puisque Ies amplitudes des deuxièmes et troisièmes harmoniques sont diminuées par la présence du fluide supérieur, le profil d'onde est plus sinusoïdal que celui de l'onde progressive en surface libre. La vitesse de propagation est également diminuée.

Une certaine modification dans la preuve de convergence se montre souhaitable, et peut être utilisée pour évaluer la gamme des amplitudes d'ondes dont l'existence a été établie rigoureusement (comme d'ailleurs pour les ondes en surface libre - voir Hunt [2]). L'auteur montre comment une modification des transformations utilisées par Levi-Civita conduit à l'évaluation de l'amplitude de l'onde maximale, bien que le résultat ainsi obtenu soit moins précis que celui donné par la méthode de Yamada [3]. 


\section{Formulation of the problem.}

We consider progressive waves which have been reduced to rest in the usual way by introducing a steady stream of magnitude equal to the wave speed and acting in the opposite direction. The flow is therefore steady and takes place in the plane $z=x+i y$. The region of the $z$-plane occupied by the upper fluid of density $\varphi^{\prime}$ is denoted by $U$ and the region occupied by the lower fluid of density $\rho$ by $L$. It is sufficient to consider a single wavelength in the $x$-direction, so we denote that part of $U$ between the ordinates $x= \pm \lambda / 2$ by $\mathrm{U}_{1}$ and the corresponding part of $\mathrm{L}$ between $x= \pm \lambda / 2$ by $\mathrm{L}_{1}$. The following notation is adopted :

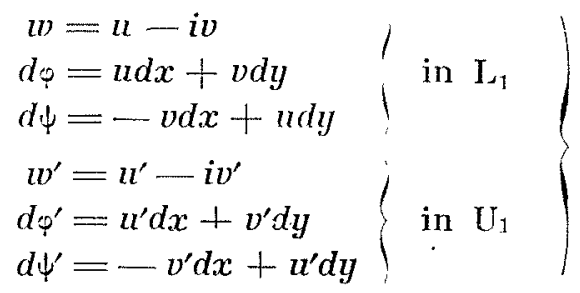

where $(u, v),\left(u^{\prime}, v^{\prime}\right)$ are components of fluid velocity and accents denote variables associated with the upper fluid of density ?'. We choose :

$$
\varphi=\psi=\varphi^{\prime}=\psi^{\prime}=0
$$

at the origin, and $\psi=\psi^{\prime}$ on the interface $l$. Further, in $\mathrm{L}_{1}$ :

$$
f=p+i
$$

and in $\mathrm{U}_{1}$

$$
f^{\prime}=\varphi^{\prime}+i \psi^{\prime}
$$

The boundary conditions to be satisfied on the interface $l$ are firstly continuity of pressure :

\section{Formulation du problème.}

Nous considérons des ondes progressives ayant été rendues stationnaires par la méthode classique, suivant laquelle on introduit un courant uni. forme, de grandeur égale à la vitesse de l'onde, et agissant dans le sens opposć à celle-ci. L'écoulement est donc permanent et se produit dans le plan $z=x+i y$. La région du plan des $z$ occupée par le fluide supérieur de densité o' est désignée par $U$, et celle occupée par le fluide inférieur de densité ? est désignée par L. Il suffit de tenir compte d'une seule longueur d'onde suivant la direction $x$, et nous désignons donc la partie de la région $U$ limitée par les ordonnées $x \pm \lambda / 2$ par $\mathrm{U}_{1}$, et la partie correspondante de la région $\mathrm{L}$, limitée par $x= \pm \zeta / 2$, par $\mathrm{L}_{1}$. Nous adoptons ensuite la notation :

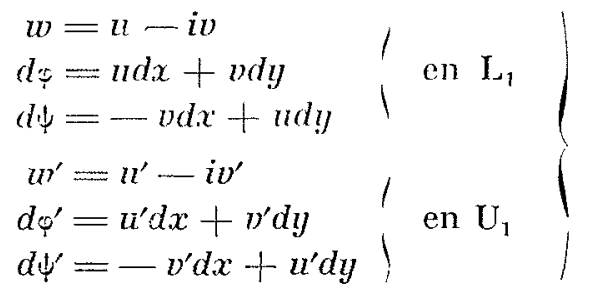

$(u, v)$ et $\left(u^{\prime}, v^{\prime}\right)$ étant des composantes de la vitesse du fluide, et les signes «prime * indiquant des variables correspondant au fluide supérieur de densité $\rho^{\prime}$.

Nous choisissons $\varphi=\psi=\varphi^{\prime}=\psi^{\prime}=0$ à l'origine, et $\psi=\psi^{\prime}$ à l'interface $l$. De plus, en $\mathrm{L}_{1}$ :

$$
w=d f / d z
$$

et en $\mathrm{U}_{1}$ :

$$
u^{\prime}=d f^{\prime} / d z
$$

Les conditions aux limites devant être satisfaites à l'interface $l$ sont, premièrement. la continuité de pression :

$$
\rho g y_{l}+\frac{1}{2} \rho|w|^{2}=\rho^{\prime} g y_{l}+\frac{1}{2} o^{\prime}\left|w w^{\prime}\right|^{2}+\mathrm{C}^{\mathrm{te}}
$$

where $y_{l}$ is the ordinate of the interface, and secondly the kinematic condition : $y_{l}$ désignant l'ordonnée de l'interface, et, deuxièmement, la condition cinématique:

$$
(v / u)_{l}=\left(v^{\prime} / u^{\prime}\right)_{l}
$$

The wave motion considered requires the rate of flow across an element of a vertical plane perpendicular to the $x$-axis to remain finite both over an arbitrary interval of time and also as either $x$ or $y$ tend to infinity. Consequently $f-c z$ is bounded in $L_{1}$ and $f^{\prime}-c z$ is bounded in $\mathrm{U}_{1}$. It follows that $\psi<0$ in $L_{1}$ and $\psi^{\prime}=0$ in $U_{1}$ with $\psi=\psi^{\prime}=0$ on $l$.
Le mouvement d'onde considéré est tel que la valeur du débit à travers un élément d'un plan vertical perpendiculaire à l'axe des $x$ reste finie, d'une part pendant un intervalle de temps arbitraire, et d'antre part, lorsque les valeurs de $x$ ou de $y$ tendent à devenir infinies. Il s'ensuit que $f-c z$ est limité en $\mathrm{I}_{1}$, el que $f^{\prime}-c z$ est limité en $U_{1}$, et de là, que $\psi<0$ en $L_{1}$, et $\psi^{\prime}>0$ en $U_{1}$, avec $\psi=\psi^{\prime}=0$ sur $l$. 


\section{Change of variables.}

The relations (2) and (3) enable the regions $L_{1}$ and $U_{1}$ to be mapped conformally onto the regions $\mathrm{L}_{1}{ }^{*}$ and $\mathrm{U}_{1}{ }^{*}$ of the $(\varphi, \psi)$ plane in which $i w^{\prime}\left(f^{\prime}\right)$ and $w(f)$ are regular, $\mathrm{U}_{1} *$ lying above the real axis and $\mathrm{L}_{1}$ * below the real axis. Differentiating (4) with respect to 9 we have :

\section{Changement des variables.}

Les relations (2) et (3) permettent une représentation conforme des régions $L_{1}$ el $U_{1}$ aux régions $\mathrm{L}_{1}{ }^{*}$ et $\mathrm{U}_{1} *$ du plan $(0, \psi)$, dans lequel $w^{\prime}\left(f^{\prime}\right)$ ct $\not r(f)$ sont réguliers, $U_{1}$ * étant siluée au-dessus de l'axe réel, et $\mathrm{L}_{1}$ * au-dessous de ce mème axe. Si nous différencions (4) par rapport à p, nous avons :

$$
2 g\left(\rho-\rho^{\prime}\right) \frac{d y_{l}}{d \varphi}=\rho^{\prime} \frac{d\left|w^{\prime}\right|^{2}}{d \varphi}-\rho \frac{d|w|^{2}}{d \varphi}
$$

on $l$, while from (2) :

sur $l$, et l'équation (2) nous donne :

$$
\frac{d y_{l}}{d \varphi}=\frac{v}{|w|^{2}}
$$

on $l$, so that :

sur $l$, de sorte que :

$$
2 g\left(\rho-\rho^{\prime}\right) \frac{v}{|w|^{2}}=\rho^{\prime} \frac{d\left|w^{\prime}\right|^{2}}{d \varphi}-\rho \frac{d|w|^{2}}{d \varphi}
$$

If we now make a change of dependent variahle :

$\left.\begin{array}{rl}\text { and } \quad \begin{array}{c}w \\ \text { and }\end{array} \quad \text { where } \omega=\theta+i \tau \\ w^{\prime}=c e^{-i \omega} \quad \text { where } \omega^{\prime}=\theta^{\prime}+i \tau^{\prime}\end{array}\right\}$

then the condition (6) at the interface takes the form :

$$
g c^{-3}\left(\rho-\rho^{\prime}\right) e^{-\tau} \sin \theta=\rho^{\prime} e^{2 \tau^{\prime}} \frac{d \tau^{\prime}}{d \varphi}-\rho e^{2 \tau} \frac{d \tau}{d \varphi}
$$

A second transformation :

Une deuxième transformation :

$$
\zeta=\exp .(-2 \pi i f / c \lambda)=\gamma \exp i \sigma
$$

now maps the region $L_{1}^{*}$ onto the interior $D_{1}$ of the circle $|\zeta|=1$ in the $\zeta-$ plane, and :

représenle la région $L_{1}$ * sur l'intérieur $D_{1} d u$ cercle $|\zeta|=1$ dans le plan $\zeta$, et :

$$
\zeta=\exp \cdot\left(-2 \pi i f^{\prime} / c \lambda\right)=\gamma \exp i \sigma
$$

maps the region $\mathrm{U}_{1} *$ onto the exterior $\mathrm{D}_{2}$ of the représente la région $\mathrm{U}_{1}{ }^{*}$ sur l'extérieur $\mathrm{D}_{2}$ du circle. From $(9)$ : cercle. D’après (9) :

$$
\frac{d \zeta}{\zeta}=-\frac{2 \pi i f}{c \lambda}=\frac{d \gamma}{\gamma}+i d \sigma
$$

so that on $|\zeta|=1, \quad d \varphi=-(c \lambda / 2 \pi) d \sigma$, and the boundary condition (8) on $|\zeta|=1$ now becomes :

$$
\frac{g \lambda}{2 \pi c^{2}}\left(\rho-\rho^{\prime}\right) e^{-\tau} \sin \theta=\rho e^{2 \tau} \frac{d \tau}{d \sigma}-\rho^{\prime} e^{2 \tau^{\prime}} \frac{d \tau^{\prime}}{d \sigma}
$$

To obtain the equation to the interface, we have from (2), (7) and (10):

Pour obtenir l'équation de l'interface, nous tirons de (2), (7) et (10):

$$
d z=\frac{d f}{w}=-\frac{\lambda}{2 \pi i} e^{i \omega} \frac{d \zeta}{\zeta}
$$


and :

et :

$$
z=-\frac{\lambda}{2 \pi i} \int e^{i \omega} \frac{d \zeta}{\zeta}
$$

In particular, on $|\zeta|==1$,

En particulier, sur $|\zeta|=1$ :

$$
z_{l}=-\frac{\lambda}{2 \pi} \int_{0}^{\sigma} e i^{\omega} d \sigma
$$

so that :

$$
\begin{aligned}
& x_{l}=-\frac{\lambda}{2 \pi} \int_{0}^{\sigma} e^{-\tau} \cos \theta d \sigma \\
& \text { and } y_{l}=-\frac{\lambda}{2 \pi} \int_{0}^{\sigma} e^{-\tau} \sin \theta d \varphi
\end{aligned}
$$

giving the implicit form of the equation to the wave profile.

Applying the change of variable (7) to the second boundary condition (5) we find that on $l$,

$$
\operatorname{tg} \theta=\operatorname{tg} \theta^{\prime}
$$

In the regions $L_{1}, U_{1}$ the principal values may be taken so that on $|\zeta|=1$,

\section{ay}
Nous pouvons preridre les valeurs principales dans les régions $L_{1}, U_{1}$ de telle sorte que, sur $|\zeta|=1:$

$$
\theta=\theta^{\prime}
$$

It will be seen that it is appropriate to assume the expansions :

$$
\omega=\sum_{1}^{\infty} \omega_{n, n^{n}} \quad \omega^{\prime}=\sum_{1}^{\infty} \omega_{n n^{\prime}}^{n}
$$

where $\mu$ is an arbitrary small parameter. Since $\omega$ is holomorphic in $D_{1}$ and $\omega^{\prime}$ is holomorphic in $D_{2}$ it follows that $\omega_{n}$ and $\omega_{n}^{\prime}$ possess the expansions:

dans lesquelles $\mu$ est un paramètre choisi arbitrairement petit. Puisque $\omega$ est holomorphe en $D_{1}$, et $\omega^{\prime}$ est holomorphe en $D_{2}$, il s'ensuit les développements de $\omega_{n}$ et $\omega_{n}^{\prime}$ :

$$
\begin{gathered}
\omega_{n}=\theta_{n}+i \tau_{n}=\sum_{1}^{\infty} c_{n, \nu} \zeta^{\nu} \\
\omega_{n}^{\prime}=\theta_{n}^{\prime}+i \tau_{n}^{\prime}=\sum_{i}^{\infty} d_{n, \nu} \zeta^{-\nu}
\end{gathered}
$$

where $c_{n, \nu}=a_{n, \nu}-i b_{n, \nu}$ and $d_{n, \nu}=e_{n, \nu}-i h_{n \nu} \quad$ où $\quad c_{n, \nu}=a_{n, \nu}-i b_{n, \nu} \quad$ et $\quad d_{n, \nu}=e_{n, \nu}-i h_{n, \nu}$

On the circle $|\zeta|=1, \zeta=e^{i \sigma}$ and so :

Sur le cercle $|\zeta|=1, \zeta=e^{i \sigma}$, d'où :

$$
\begin{aligned}
0_{n} & =\sum_{1}^{\infty}\left(a_{n, \nu} \cos v \sigma+b_{n, \nu} \sin v \sigma\right), & \tau_{n} & =\sum_{1}^{\infty}\left(a_{n, \nu} \sin v \sigma-b_{n, \nu} \cos v \sigma\right) \\
\theta_{n}^{\prime} & =\sum_{1}^{\infty}\left(e_{n, \nu} \cos v \sigma-h_{n, \nu} \sin v \sigma\right), & \tau_{n}^{\prime} & =\sum_{1}^{\infty}\left(-e_{n, \nu} \sin v \sigma-h_{n, \nu} \cos v \sigma\right)
\end{aligned}
$$

From (13) it follows that $\theta_{n}=\theta_{n}^{\prime}$ for all $\sigma$ on $\zeta=e^{i \sigma}$ thus :

Il découle de (13) que $\theta_{n}=\theta^{\prime}{ }_{n}$ pour tous les $\sigma$ en $\zeta=e^{i \sigma}$, d'où :

$$
a_{n, \nu}=e_{n, \nu} \quad b_{n, \nu}=-h_{n, \nu} \quad \text { and } \tau_{n}=-\tau_{n}^{\prime} \quad a_{n, \nu}=e_{n, \nu} \quad b_{n, \nu}=-h_{n, \nu} \quad \text { et } \tau_{n}=-\tau_{n}^{\prime}
$$

and so on $|\zeta|=1$,

et ainsi, sur $|\zeta|=1$,

$$
\tau=-\tau^{\prime}
$$


Substituting from (13) and (17) in (11), the boundary condition becomes :
En faisant intervenir (13) et (17) en (11), la condition à la limite devient :

$$
\frac{g \lambda}{2 \pi c^{2}}\left(\varphi \cdot-\rho^{\prime}\right) e^{-\tau} \sin 0=\rho e^{2 \tau} \frac{d \sigma}{d \sigma}+\rho^{\prime} e^{-2 \tau} \frac{d \tau}{d \sigma}
$$

\section{General form of solution.}

If we neglect terms of order higher than the first in $0, \tau,(18)$ takes the form :

\section{Forme de solution générale.}

Si nous négligeons les termes d'un ordre supérieur au premier terme en $0, \tau$ (18) prend la forme :

$$
\frac{g \lambda}{2 \pi c^{2}}\left(\varphi-\rho^{\prime}\right) \theta=\left(\varphi+\rho^{\prime}\right) \frac{d \tau}{d \sigma}
$$

Clearly, et, évidemment :

$$
\frac{d \tau}{d \sigma}=\mathcal{R}\left(\frac{d \omega}{i d \sigma}\right)=\mathscr{R}\left(\zeta \frac{d \omega}{d \zeta}\right)
$$

and $0=\mathcal{R}(\omega)$. Since $\omega(0)=0$,

$\zeta \frac{d \omega}{d \zeta}-q \omega=0 \quad$ where $q=\frac{g \lambda}{2 \pi c^{2}}\left(\frac{\rho-\rho^{\prime}}{\rho+\rho^{\prime}}\right)$

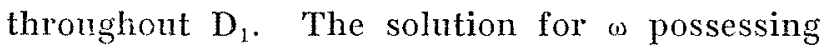
the required properties in $\mathrm{D}_{1}$ is $\omega=-i \mu \zeta^{\prime \prime}$ where $q=1$, so that the first order solution is given by :

$$
\omega=-i \mu \zeta \quad \text { and } c^{2}=\frac{g \lambda}{2 \pi}\left(\frac{\rho-\rho^{\prime}}{\rho+\rho^{\prime}}\right)
$$

which is the solution given by Lamb [4]. From (9) we have :

$$
\theta_{1}=\sin \sigma \quad \text { and } \tau_{1}=-\cos \sigma
$$

The functions $\omega_{n}$ must be holomorphic in $D_{1}$ and we may assert that $\omega-\omega_{1} \omega_{1}$ contains $\zeta^{2}$ as a factor by suitable choice of the parameter $\mu$.

There are several forms in which (18) may be solved by successive approximation, but that which shows the similarity with Levi-Civita's equation is obtained by writing : et $0=\mathcal{R}(\omega)$. Puisque $\omega(0)=0$,

$$
\zeta \frac{d \omega}{d \zeta}-q \omega=0 \quad \text { où } q=\frac{g \lambda}{2 \pi c^{2}}\left(\frac{\rho-\rho^{\prime}}{\rho+\rho^{\prime}}\right)
$$

partout en $D_{1}$. La solution pour $\omega$ ayant les caractéristiques désirées en $\mathrm{D}_{1}$ est $\omega=-i$ iu 'q où $q=1$, et la solution au premier ordre est donc donnce par :

$$
\omega=-i_{i} \zeta \quad \text { et } c^{2}=\frac{g \lambda}{2 \pi}\left(\frac{o-\rho^{\prime}}{\rho+\rho^{\prime}}\right)
$$

laquelle est la solution donnée par Lamb [4]. Nous avons, à partir de (9):

$$
\theta_{1}=\sin \sigma \quad \text { et } \tau_{1}=-\cos \sigma
$$

Les fonctions $\omega_{n}$ doivent être holomorphes en $D_{1}$, et nous pouvons affirmer que $\omega-\mu \omega_{1}$ contient $\zeta^{2}$ comme facteur, on choisissant une valeur convenable du paramètre u. (18) peut être résolue sous plusieurs formes par approximations successives, mais celle qui démontre l'analogie avec l'équation de revi-Civita s'obtient en posant :

$$
\begin{array}{ccc}
\frac{d \tau}{d \sigma}-0 & =(1-k) \mathrm{P}(0, \tau)-k \theta+\frac{\rho^{\prime}}{\rho+\rho^{\prime}} \frac{d}{d \sigma} \mathrm{R}(\tau) \\
\text { where } \mathrm{P}(\theta, \tau)=e^{-3 \tau} \sin \theta-\theta, & \text { où } \mathrm{P}(0, \tau)=e^{-3 \tau} \sin 0-0, \\
\mathrm{R}(\tau)=\frac{1}{4} e^{-4 \tau+\tau,} \quad q=1-k & \mathrm{R}(\tau)=\frac{1}{4} e^{-4 \tau+\tau,} \quad q=1-k
\end{array}
$$

With :

Pour :
$0=\sum_{1}^{\infty} 0_{n} \mu^{n}$
$\tau=\sum_{1}^{\infty} \tau_{n} \Lambda^{n}$
and $k=\sum_{1}^{\infty} k_{n} \mu^{n}$,
$0=\sum_{1}^{\infty} 0_{n} u^{n}$.
$\tau=\sum_{1}^{\infty} \tau_{n} \mu^{n}$
et $k=\sum_{i}^{\infty} k_{n} \mu^{n}$, 
it is clear that the coefficient of $\mu^{n}$ in the function : il est évident que le coefficient de $\mu^{n}$ dans la fonction :

$$
(1-k)\left(e^{-3 \tau} \sin \theta-\theta\right)-\frac{\rho^{\prime}}{\rho+\rho^{\prime}}\left(e^{-4 \tau}-1\right) \frac{d \tau}{d \sigma}
$$

depends only on the functions :

$$
0_{1} \ldots 0_{n-1}, \tau_{1} \ldots \tau_{n-1}, k_{1} \ldots k_{n-2} .
$$

We now assume that these functions are known and have the properties :

(i) $k_{1}=k_{3} \ldots=0$ up to $k_{n-2}$.

(ii) $\omega_{\nu}$ is a polynomial of degree ${ }_{\nu}$ divisible by $\zeta^{2}$ such that

(a) it is purely imaginary when $\zeta$ is real,

(b) $\mu^{\nu} \omega_{\nu}$ is unchanged when writing $-\zeta$ and - $y$ for $\zeta, p$.

It then follows that on $|\zeta|=1, \theta_{1} \ldots \theta_{n-1}$ are odd functions of $\sigma, \tau_{1} \ldots \tau_{t-1}$ are even functions of $\sigma$, and $\mu^{\nu} \theta_{\nu}, \mu^{j /} \tau_{\nu}$ are unaltered on changing $\sigma$ to $\sigma+\pi$ and $\mu$ to $-\mu$. Thus up to the order $\mu_{n}$ inclusive $(1-k) \mathrm{P}(\theta, \tau)$ is an odd function of $\theta$ therefore of $\sigma$,

and : dépend uniquement des fonctions $\theta_{1} \ldots \theta_{n-1}$, $\tau_{1} \ldots \tau_{n-1}, k_{1} \ldots k_{n-2}$. Nous admettons que ces fonctions sont connues, et qu'elles possèdent les caractéristiques :

(i) $k_{1}{ }^{\prime}=k_{3} \ldots=0$ jusqu'à $k_{n-2}$.

(ii) $\omega_{y}$, est un polynôme de degré $\nu$, divisible par $\zeta^{2}$, de telle manière que :

a) Sa valeur est purement imaginaire lorsque $\zeta$ est réel,

b) $\mu^{1} \omega_{y}$ ne varie pas lorsqu'on pose $-\zeta$ et $-\mu$ au lieu de $\zeta, \ldots$.

Il s'ensuit alors que sur $|\zeta|=1, \theta \ldots \theta_{n-1}$ sont des fonctions impaires de $\sigma$, que $\tau_{1} \ldots \tau_{n-1}$ sont des fonctions paires de $\sigma$, et que $\mu^{\nu} \theta_{\nu}, \mu^{\nu} \tau_{\nu}$ sont inchangés lorsqu'on fait varier $\sigma$ jusqu'à $\sigma+\pi$, et $\mu$ jusqu'à - $-\mu$. Ainsi, jusqu'à et y compris l'ordre $k^{n},(\mathbf{1}-k) \mathrm{P}(\theta, \tau)$ est une fonction impaire de $\theta$, et donc également de $\sigma$, et :

$$
\mathrm{Q}_{n}=\frac{1}{n !}\left[\frac{d^{n}}{d u^{n}}(1-k) \mathrm{P}\right]_{\mu=0}
$$

is also an odd function of $\sigma$. Since $k$ by hypothesis contains only even powers of $\mu$, then $(1-k) \mathrm{P}$ is unaltered on writing $\sigma+\pi$ and $-\mu$ for $\sigma$ and $\mu$. Thus, est également une fonction impaire de $\sigma$. Puisque, d'après l'hypothèse, $k$ ne contient que des puissances paires de $\mu$, il s'ensuit que $(1-k) \mathrm{P}$ ne varie pas lorsqu'on pose $\sigma+\pi$ et $-\mu$ au lieu de $\sigma$ et $\mu$. Ainsi,

$$
\begin{aligned}
Q_{n}(-\sigma)=-Q_{n}(\sigma), & \\
\text { and } Q_{n}(\sigma+\pi)=(-1)^{n} Q_{n}(\sigma) . & \text { et } Q_{n}(\sigma+\pi)=(-1)^{n} Q_{n}(\sigma) .
\end{aligned}
$$

Since the functions $\theta_{\nu}, \tau_{y}, \nu=1,2, \ldots n-1$, which form $P(\theta, \tau)$ may be regarded as polynomials in $\cos \sigma$ and $\sin \sigma$ on $|\xi|=1$, we may write:

Puisque les fonctions :

$$
\theta_{\nu}, \tau_{\nu}, \nu=1,2, \ldots n-1 \text {, }
$$

constituant $\mathbf{P}(\theta, \tau)$ peuvent être considérées comme étant des polynômes en $\cos \sigma$ et $\sin \sigma$ sur $|\zeta|=1$, nous pouvons écrire :

$$
\mathrm{Q}_{n}=\sum_{\nu=6}^{[n / 2]} q_{n, n-2 \nu} * \sin (n-2 \nu) \sigma
$$

By similar arguments we find :

Nous trouvons, par des considérations analogues :

$$
\mathrm{R}_{n}=\sum_{\nu=0}^{[n / 2]} q_{n, n-2 \nu}^{\prime} \cos (n-2,) \sigma+1
$$

The coefficient of $y^{n}$ in $k \theta$ is :

$$
k_{1} \theta_{n-1}+k_{2} \theta_{n-2}+\ldots k_{n-1} \theta_{1}
$$

Le coefficient de $\mu^{\text {sl }}$ en $k \theta$ est :

$$
k_{1} \theta_{n-1}+k_{2} \theta_{n-2}+\ldots k_{n-1} \theta_{1}
$$


and writing :

et en posant :

$$
\mathrm{X}_{n}(\sigma)=\mathrm{Q}_{n}(\sigma)--\sum_{\nu=1}^{n-2} k_{\nu} \theta_{n-\nu}
$$

which is clearly of the same form as $Q_{n}(\sigma)$, we have :

dont la forme est manifestement analogue à $Q_{n}(\sigma)$, nous avons :

$$
\mathrm{X}_{n}=\sum_{\nu=0}^{\left[n_{1} L_{1}\right.} q_{n, n-2 \nu} \sin (n-2 \nu) \sigma
$$

Equating coefficients of $\mu^{n}$ in (21) and substituting the expansions (23), (24), we have:
La mise en équation des coefficiens de $\mu^{n}$ dans (21), et l'introduction des développements (23), (24), nous donnent :

$$
\frac{d \tau_{n}}{d \sigma}-\theta_{n}=\sum_{\nu=0}^{(n / 2)-1}\left\{q_{n, n-2 \nu}-\frac{\rho^{\prime}(n-2 \nu)}{\rho+\rho^{\prime}} q_{n, n-2 \nu}^{\prime}\right\} \sin (n-2 \nu) \sigma+\left\{q_{n, 1}-\frac{\rho^{\prime} q_{n, 1}^{\prime}}{\rho+\rho^{\prime}}-k_{n-1}\right\} \sin \sigma
$$

Further, from (16),

En outre, nous tirons de (16):

$$
\frac{d \tau_{n}}{d \sigma}-\theta_{n}=\sum_{2}^{\infty}\left\{(\nu-1) a_{n, \nu} \cos v \sigma+(\nu-1) b_{n, \nu} \sin v \sigma\right\}
$$

so that substituting in (25) and equating harmonics in $\sigma$,

$$
\begin{aligned}
& q_{n, 1}-\frac{\rho^{\prime} q_{n, 1}^{\prime}}{\rho+\rho^{\prime}}=k_{n-1}, \quad a_{n \nu}=0 \\
& b_{n, \nu}=\frac{q_{n, n-2 \nu}}{n-2 \nu-1}-\frac{\rho^{\prime}(n-2 \nu)}{\left(\varphi+\rho^{\prime}\right)(n-2 \nu-1)} q_{n, n-2 n}^{\prime} \\
& \text { for } y \leqslant[n / 2\rceil-1 \\
& q_{n, 1}-\frac{\varphi^{\prime} q_{n, 1}^{\prime}}{\varphi+\varphi^{\prime}}=k_{n-1}, \quad a_{n \nu}=0 \\
& \text { pour } \vee \leqslant[n / 2]-1
\end{aligned}
$$

de sorte que, en substituant en (25), et en égalisant les harmoniques en $\sigma$ :

pour tous les

from which, using (15), (23) and (24), we deduce

that:

d'où, en utilisant (15), (23) et (24), nous déduisons que :

$$
\omega_{n}(\zeta)=-{ }_{\nu=0}^{(n / 2)} \sum_{\nu=0}^{-1} \frac{\left(\rho+\varphi^{\prime}\right) q_{n, n-2 \nu}-\varphi^{\prime}(n-2 \nu) q_{n, n-2 \nu}^{\prime} \zeta^{n-2 \nu}}{\left(\rho+\rho^{\prime}\right)(n-2 \nu-1)}
$$

and :

et :

$$
k_{n-1}=\frac{1}{\pi} \int_{-\pi}^{\pi}\left\{\mathrm{X}_{n}(\sigma)-\frac{\sigma^{\prime}}{\rho+\rho^{\prime}}-\frac{d}{d \sigma} \mathrm{R}_{n}(\sigma)\right\} \sin \sigma d \sigma
$$

Inspection of the expansions (23), (24) show that these functions possess the properties ascribed to $\omega_{2}(\zeta) \ldots \omega_{n-1}(\zeta)$ and $k_{n-2}$. Since these properties are easily verified for $\omega_{2}(\zeta)$ and $k_{2}$, the general result follows by induction.

\section{Convergence.}

As in Levi-Civita's method we define the auxiliary function :
L'examen des développements (23) (24) montre que ces fonctions possèdent les caractéristiques assignées à $\omega_{2}(\zeta) \ldots\left(\omega_{n-1}(\zeta)\right.$ et $k_{n-2}$ Etant donné que ces caractéristiques sont aisément vérifiées pour $\omega_{2}(\zeta)$ et $k_{2}$, le résultat général suit par induction.

\section{Convergence.}

De même que dans la méthode de Levi-Civita, nous définissons la fonction auxiliaire :

$$
N(\zeta)=\zeta \log (1-\zeta)^{-1}
$$


then it may be shown that if:

on peut alors montrer que, si :

$$
\mathrm{S}_{n}(\sigma)=\frac{d}{d \sigma} \mathbf{R}_{n}(\sigma)
$$

then :

alors :

$$
\omega_{n}=\frac{1}{\pi} \int_{-\pi}^{\pi} \mathrm{N}\left(\zeta e^{-i \sigma_{1}}\right)\left\{\mathrm{X}_{n}\left(\sigma_{1}\right)-\frac{\rho^{\prime}}{\rho+\rho^{\prime}} \mathrm{S}_{n}\left(\sigma_{1}\right)\right\} d \sigma_{1}
$$

and :

et :

$$
\frac{d \omega_{n}}{d \zeta}=\frac{1}{\pi i \zeta} \int_{-\pi}^{\pi} N\left(\zeta e^{-i \sigma_{1}}\right)\left\{\mathrm{X}_{n}^{\prime}\left(\sigma_{1}\right)-\frac{\rho^{\prime}}{\rho+\varphi^{\prime}} \mathrm{S}_{n}^{\prime}\left(\sigma_{1}\right)\right\} d \sigma_{1}
$$

Consequently upper bounds may be assigned of the form :

$$
\left|\omega_{n}\right| \leqslant J M_{n} \quad \text { and }\left|d \omega_{n} / d \zeta\right| \leqslant J M_{n}^{\prime}
$$

where $M_{n}$ and $M_{n}^{\prime}$ are the maximum absolute values on $|\zeta|=1$ of :

$$
\mathrm{X}_{n}-\frac{\rho^{\prime}}{\rho+\rho^{\prime}} \mathrm{S}_{n} \quad \text { and } \mathrm{X}_{n}^{\prime} \cdot-\frac{\rho^{\prime}}{\rho-\frac{1}{1} \rho^{\prime}} \mathrm{S}_{n}^{\prime}
$$

and $J$ is a constant which is not less than the maximum value within and on $|\zeta|=1$ of the integral :

$$
\frac{1}{\pi} \int_{-\pi}^{\pi}\left|\log \left(1-\zeta e^{-i v_{1}}\right)-1\right| d \sigma_{1}
$$

Levi-Civita found that $\mathrm{J} \leqslant 2.6449$, but refined inequalities and a modification of $N|\zeta|$ lead to the improved estimate $J \leqslant 1.2825$. It is not convenient to apply "Stokes majorant functions" to this problem to establish convergence, but to use a simpler majorant function used by LeviCivita in an earlier paper [5]. We suppose that $\left|\theta_{n}\right|,\left|\tau_{n}\right|,\left|d \theta_{n} / d \sigma\right|$ and $\left|d \tau_{n} / d \sigma\right|$ are bounded by $\Omega_{n}$ and $k$ by $K$, where :

Par conséquent, nous pouvons fixer des limites supérieures, de la forme :

$$
\left|\omega_{n}\right| \leqslant \mathrm{JM}_{n} \quad \text { et }\left|d \omega_{n} / d \zeta\right| \leqslant \mathrm{JM}_{n}^{\prime}
$$

$\mathrm{M}_{n} \mathrm{M}_{n}^{\prime}$ étant les valeurs absolues maximales, sur $|\zeta|=1$, de :

$$
\mathrm{X}_{n}-\frac{\rho^{\prime}}{\rho+\rho^{\prime}} \mathrm{S}_{n} \quad \text { et de } \mathrm{X}_{n}^{\prime}-\frac{\rho^{\prime}}{\rho+\rho^{\prime}} \mathrm{S}_{n}^{\prime}
$$

ct J étant une constante, dont la valeur n'est pas inférieure à la valeur nominale dans et sur $|\zeta|=1$, de l'intégrale :

Levi-Civita a trouvé $J \leqslant 2,6449$; toutefois, en raf finant les inégalités, et en modifiant $\mathrm{N}(\zeta)$, on parvient à une meilleure évaluation, soit :

$$
J \leqslant 1,2825 \text {. }
$$

Les « fonctions majorantes 》 de Stolies ne conviennent pas pour démontrer la convergence dans ce problème; on applique plus avantageusement une fonction majorante plus simple, utilisée par Levi-Civita dans un mémoire antérieur [5]. Nous supposons que $\left|\hat{\theta}_{n}\right|,\left|\tau_{n}\right|,\left|d 0_{n} / d \sigma\right|$ et $\left|d \tau_{n} / d \sigma\right|$ sont délimités par $\Omega_{n}$, et que $k$ est délimité par $K$, avec :

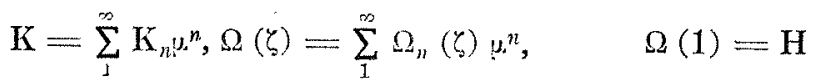

The functions :

$$
\mathrm{X}_{n}(\sigma)-k_{n-1} \sin \sigma \text { and } \sigma^{\prime} \mathrm{S}_{n}(\sigma) /\left(\rho+\rho^{\prime}\right)
$$

are bounded by :

$$
\begin{aligned}
& \frac{1}{n !}\left[\frac{d^{n}}{d \mu^{n}}\{(1+\mathrm{K}) \mathrm{G}(\Omega)+\mathrm{K} \Omega\}\right]_{\mu=0} \\
& \text { and } \frac{\rho^{\prime}}{\rho+\rho^{\prime}} \frac{1}{n !}\left[\frac{d^{n}}{d \mu^{n}}\left\{\Omega\left(e^{4 \Omega}-1\right)\right\}\right]_{\mu=0}
\end{aligned}
$$

Les fonctions :

$$
\mathrm{X}_{n}(\sigma)-k_{n-1} \sin \sigma \text { et } \rho^{\prime} \mathrm{S}_{n}(\sigma) /\left(\rho+\rho^{\prime}\right)
$$

sont délimitées par :

$$
\begin{aligned}
& \frac{1}{n !}\left[\frac{d^{n}}{d \omega^{n}}\{(1-\mathrm{K}) \mathrm{G}(\Omega)+\mathrm{K} \Omega\}\right]_{\mu=0} \\
& \text { et } \frac{\rho}{\varphi+\rho^{\prime}} \frac{1}{n !}\left[\frac{d^{n}}{d \varphi^{n}}\left\{\Omega\left(e^{4 \Omega}-1\right)\right\}\right]_{\mu=0}
\end{aligned}
$$


where $G(\Omega)=e^{3 \Omega} \sinh \Omega-\Omega$. It follows that from the first of (29): où $G(\Omega)=e^{3 \Omega} \sinh \Omega-\Omega$. Il s'ensuit qu'à partir du premier terme de (29):

$$
\Omega_{n}(\zeta)=\frac{J}{n !}\left[\frac{d_{n}}{d_{\mu}{ }^{n}}\left\{(1+\mathrm{K}) \mathrm{G}(\Omega)+\mathrm{K} \Omega+\frac{\rho^{\prime}}{\rho+\rho^{\prime}} \Omega\left(e^{4 \Omega}-1\right)\right\}\right]_{\mu=0}
$$

Multiplying by $\mu^{n}$ and summing over $n$, using the expansion for $\Omega$ and putting $\zeta=1$, we have :
En multipliant par u $^{n}$ et en opérant la sommation sur $n$, en utilisant le développement de $\Omega$, et en posant $\zeta=1$, nous obtenons :

$$
\mathrm{H}=\mu+\mathrm{J}\left[(1+\mathrm{K}) \mathrm{G}(\mathrm{H})+\mathrm{KH}+\frac{\rho^{\prime}}{\varphi+\varphi} \mathrm{H}\left(e^{4 \mathrm{H}}-1\right)\right]
$$

Similarly from (28) :

De même, nous obtenons, à partir de (28) :

$$
\mathrm{K}=\frac{2}{p}\left[(1+\mathrm{K}) \mathrm{G}(\mathrm{H})+\frac{\rho^{\prime}}{\rho+\rho} \mathrm{H}\left(e^{4 \mathrm{H}}-1\right)\right]
$$

Equations (30) and (31) define $H$ and $K$ as holomorphic functions of for for some finite range in the neighbourhood of $\mu_{c}=0$. The series (14) are then uniformly convergent together with $d \omega / d \zeta$, $d \omega^{\prime} / d \zeta$ in this range. The range of permitted wave amplitudes given by (30) (31) is a function of the parameter $\rho^{\prime} / 0$, but as in the case of waves at a free surface [2], the apparent maximum amplitude given by this convergence proof is far smaller than that known to exist in reality.

Another method of estimating the highest wave amplitude is to use an alternative change of dependent variable. It is evident from (7) that at a wave crest where $w=0, \tau$ must diverge. We therefore choose a form in which a known finite value gives $w=0$, for example :

Les équations (30) et (31) définissent $\mathrm{H}$ et $\mathrm{K}$ comme étant des fonctions holomorphes de $\mu$ pour une zone finie quelconque autour de $\mu=0$. Les séries (14) sont alors uniformément convergentes, ainsi que $d \omega / d \zeta, d \omega^{\prime} / d \zeta$ dans cette zone. La gamme des amplitudes d'onde admises donnée par (30), (31) est une fonction du paramètre

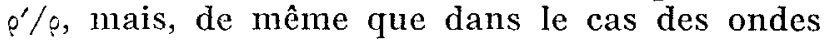
en surface libre [2], l'amplitude maximale apparente donnce par cette preuve de convergence est beaucoup plus faible que celle que l'on sait exister dans la réalité.

Une autre méthode pour évaluer l'amplitude d'onde maximale consiste à choisir un autre changement de variable. Il est évident d'après (7) que $\tau$ doit être divergent, pour une crête d'onde correspondant à $w=0$. Nous choisirons donc une forme dans laquelle une valeur finie connue donne $w=0$, par exemple :

$$
w=c\left(\cos \omega^{(1)}-\sin \omega(1)\right)
$$

so that $u=v=0$ when $\theta^{(1)}=\pi / 4, \tau^{(1)}=0$. To the fourth order, when $\rho^{\prime}=0$,

de sorte que $u=v=0$, lorsque $\theta^{(1)}=\pi / 4$, $\tau^{(1)}=0$. Au quatrième ordre, lorsque $\rho^{\prime}=0$ :

$$
\omega^{(1)}=\mu^{\zeta}+\frac{1}{2} \mu^{2} \zeta^{2}+\frac{7}{6} \mu^{3} \zeta^{3}+\mu^{4}\left(\frac{5}{3} \zeta^{4}+\zeta^{2}\right) \ldots \ldots
$$

and for the highest crest $(\zeta=1)$,

et, pour la crête d'onde maximale $(\zeta=1)$,

$$
\theta(1)=\frac{\pi}{4}=\mu+\frac{1}{2} \mu^{2}+\frac{7}{6} \mu^{3}+\frac{8}{3} \mu^{4}
$$

from which :

$$
(h / \lambda)_{\max }<1 / 5.36
$$

Various other transformalions similar to (32) have failed to improve substantially on this estimate which cxceeds Michell's ratio $1 / 7$ which has been confirmed by Yamada [3]. 'The superiority of Yamada's method is not surprising in d'où :

$$
(h / \lambda)_{\max }<1 / 5,36
$$

II n'a suère été possible, au moyen d'autres transformations analogues à (32), d'amćliorer d'une manière appréciable quelconque cette évaluation, laquelle dépasse le rapport 1/7 énoncé par Milchell et confirmé par Yamada [3]. La 
view of the nature of the singularity at the wave crest.

\section{Third order solution.}

Repeated application of (27) and (28) lead to the following results up to the third order.

$$
\begin{aligned}
& \omega_{1}=-i \zeta, \\
& \omega_{3}=-i \frac{17 \rho\left(\rho-\rho^{\prime}\right)+2 \rho^{\prime 2}}{6\left(\rho+\rho^{\prime}\right)^{2}} \zeta^{3},
\end{aligned}
$$

On evaluating $e^{i \omega}$ and using (12) we have parametric forms of the wave profile equation, which, on elimination of the parameter $\sigma$, lead to the result : supériorité de la méthode de Yamada ne surprend guère, étant donné la nature de la singularité à la crête d'onde.

\section{Solution au troisième ordre.}

L'application répétée de (27) et (28) conduit aux résultats suivants, jusqu'au troisième ordre :

$$
\begin{aligned}
& \omega_{2}=-i \frac{3 \rho-\rho^{\prime}}{2\left(\rho+\rho^{\prime}\right)} \zeta^{2}, \\
& k_{2}=\frac{\rho^{2}-3 \rho \rho^{\prime}}{\left(\rho+\rho^{\prime}\right)^{2}}
\end{aligned}
$$

En évaluant $e^{i \omega}$ et en utilisant (12), nous obtenons des formes paramétriques de l'équation du profil d'onde, lesquelles, une fois éliminé le paramètre $\sigma$, conduisent au résultat suivant :

$$
y_{i}=\frac{\lambda}{2 \pi}\left\{a \cos m x+\frac{1}{2} a^{2}\left(\frac{\rho-\rho^{\prime}}{\rho+\varphi^{\prime}}\right) \cos 2 m x+a^{3} \frac{3 \varphi^{2}-10 \rho o^{\prime}+3 \rho^{\prime 2}}{8\left(\rho+\varphi^{\prime}\right)^{2}} \cos 3 m x \ldots\right\}
$$

where

où :

$$
m=2 \pi / \lambda, \quad a=\mu+3 \mu \mu^{3} \frac{3 \rho-\rho^{\prime}}{8\left(\rho+\rho^{\prime}\right)}
$$

Using $q=1-k$, the velocity of propagation is given to the third order by :
Avec $q=1-k$, la vitesse de propagation est donnée, au troisième ordre, par :

$$
c^{2}=\frac{9}{m}\left(\frac{\rho-\rho^{\prime}}{\rho+\rho^{\prime}}\right)\left\{1+\frac{\rho^{2}+\rho^{\prime 2}}{\left(\rho+\rho^{\prime}\right)^{2}} m^{2} a^{2}\right\}
$$

It is evident that the amplitude of the second and third harmonics are reduced by the presence of the upper fluid of density $\hat{r}^{\prime}$.

When the two fluids are contained between rigid horizontal planes $y=h^{\prime}, y=-h$, it is simpler to solve for the wave profile by a power series expansion of the Stokes-Rayleigh type. With the notation :

$$
\begin{array}{r}
\mathrm{S}_{n}=\sinh n m h, \mathrm{~S}_{n}^{\prime}=\sinh n m h^{\prime}, \\
\mathrm{C}_{n}=\cosh n m h, \mathrm{C}_{n}^{\prime}=\cosh n m h^{\prime}
\end{array}
$$

the wave profile to this order is :
Il est évident que les amplitudes des deuxième et troisième harmoniques sont réduites par la présence du fluide supérieur de densité ?'

Lorsque les deux fluides sont contenus entre des plans horizontaux rigides $y=h^{\prime}, y=-h$, la solution pour le profil d'onde s'obtient plus aisément par le développement en série, du type Stokes-Rayleigh. Avec les notations :

$$
\begin{aligned}
& \mathrm{S}_{n}=\sinh n m h, \quad \mathrm{~S}_{n}^{\prime}=\sinh n m h^{\prime}, \\
& \mathrm{C}_{n}=\cosh n m h, \quad \mathrm{C}_{n}^{\prime}=\cosh n m h^{\prime},
\end{aligned}
$$

le profil d'onde, à cet ordre, est :

$$
y=a_{1} \cos m x+a_{2} \cos 2 m x+a_{3} \cos 3 m x,
$$

where

avec :

$$
\begin{aligned}
& \left(\rho \frac{\mathrm{S}_{1}}{\mathrm{C}_{1}}+\rho^{\prime} \frac{\mathrm{S}_{1}^{\prime}}{\mathrm{C}_{1}^{\prime}}\right) a_{2}=\frac{1}{2} m a_{1}^{2}\left[\frac{\rho \mathrm{C}_{1}\left(\mathrm{C}_{2}+2\right)}{\mathrm{S}_{1} \mathrm{~S}_{2}}-\frac{\rho^{\prime} \mathrm{C}_{1}^{\prime}\left(\mathrm{C}_{z}^{\prime}+2\right)}{\mathrm{S}_{1}^{\prime} \mathrm{S}_{2}^{\prime}}\right] \\
& \left(? \frac{\mathrm{C}_{1} \mathrm{~S}_{1}^{2}}{\mathrm{~S}_{3}}+\rho^{\prime} \frac{\mathrm{C}_{1}^{\prime} \mathrm{S}_{1}^{\prime}}{\mathrm{S}_{3}^{\prime}}\right) a_{3}=\frac{m a_{1} a_{2}}{16}\left[\frac{?\left(2 \mathrm{C}_{4}+3 \mathrm{C}_{2}+4\right)}{\mathrm{S}_{1}^{2}\left(2 \mathrm{C}_{2}+1\right)}-\frac{\rho^{\prime}\left(2 \mathrm{C}_{4}^{\prime}+3 \mathrm{C}_{2}^{\prime}+4\right)}{\mathrm{S}_{1}^{\prime}\left(2 \mathrm{C}_{2}^{\prime}+1\right)}\right] \\
& \ldots . . \frac{m a_{1}^{3}}{16}\left[\frac{\rho \mathrm{C}_{1}^{3}\left(\mathrm{C}_{2}+2\right)}{\mathrm{S}_{1}^{3}\left(2 \mathrm{C}_{2}+1\right)}+\frac{\rho^{\prime} \mathrm{C}_{1}^{\prime}\left(\mathrm{C}_{2}^{\prime}+2\right)}{\mathrm{S}_{1}^{\prime}\left(2 \mathrm{C}_{2}^{\prime}+1\right)}\right]
\end{aligned}
$$


The velocity of propagation is given by the relation :

$$
\begin{aligned}
& c^{2}=\frac{9\left(\rho-\rho^{\prime}\right)}{m\left(\rho \frac{\mathrm{C}_{1}}{\mathrm{~S}_{1}}+\rho^{\prime} \frac{\mathrm{C}_{1}^{\prime}}{\mathrm{S}_{1}^{\prime}}\right)} \times \\
& \quad \times\left\{1+\frac{m^{2} a_{1}^{2}\left(\frac{\rho \mathrm{C}_{1}\left(\mathrm{C}_{1}^{2}-2\right)}{\mathrm{S}_{1}^{3}}+\frac{\rho^{\prime} \mathrm{C}_{1}^{\prime}\left(\mathrm{C}_{1}^{2}-2\right)}{\mathrm{S}_{1}^{\prime}}\right)-m a_{2}\left(\frac{\rho\left(2 \mathrm{C}_{1}^{2}+1\right)}{\mathrm{S}_{1}^{2}}-\frac{\rho^{\prime}\left(2 \mathrm{C}_{1}^{2}+1\right)}{\mathrm{S}_{1}^{\prime 2}}\right)}{2\left(\rho \frac{\mathrm{C}_{1}}{\mathrm{~S}_{1}}+\rho^{\prime} \frac{\mathrm{C}_{1}^{\prime}}{\mathrm{S}_{1}^{\prime}}\right)}\right\}
\end{aligned}
$$

These results readily reduce to $(34),(35)$ as $h, h^{\prime} \rightarrow \infty$, or, if we put $z^{\prime}=0$, to the StokesStruik solution for progressive waves in a fluid of finite depth $h$.
La vitesse de propagation est donnée par la relation :

\section{Bibliographie}

[1] Levi-Civita, T. (1925). - La détermination rigoureuse des ondes permanentes d'ampleur finic. Math. Ann., 93, 264-314.

[2] Hunt, J.N. (1953). - A note on gravity waves of finite amplitude. $Q$. J. Mech. and Appl. Math., 6, 336-343.

[3] YaMADA, H. (1957). - Highest waves of permanent
Ces résultats se réduisent facilement à (34) et (35) lorsque $h, h^{\prime} \rightarrow \infty$, ou bien, si nous posons $\rho^{\prime}=0$, à la solution Stokes-Struik pour les ondes progressives dans un fluide de profondeur finie $h$. type on the surlace of deep water. Rep. Res. Inst. for App. Mech., 5, 37-52.

[4] LaMs, H. - Hydrodynamies, (1932), C.U.P., 370.

[5] I.evi-Crvita, T. (1921). - Rizolizione dell'equazione funzionale che caratterizza le onde periodiche in un canale molto profondo. Math. Ann., 85, 256-278. 


\section{II. - STATIONARY OSCILLATIONS}

\section{II. - OSCILLATIONS STATIONNAIRES.}

\begin{abstract}
Stationary oscillations at a fluid interface are examined, the frequency of oscillation and the equation to the wave profile being found to the fourlh order. The method is that used by Penney and Price for oscillations at a free surface. As in the case of progressive waves, the amplitudes of the higher order harmonics are reduced by the presence of the upper fuid giving a more sinusoidal profile, and the period of oscillation is increased. It is pointed out that indeterminate harmonics arise in the profile equation.
\end{abstract}

\section{Formulation of the problem.}

We choose an origin of coordinates 0 at the interface between two semi-infinite fluids, the upper of density $\rho^{\prime}$, the lower of density $\rho$, with the axis $\mathrm{O} y$ vertically upwards. The motion is determined by velocity potentials $\varphi$, $\varphi^{\prime}$ where :

$$
\nabla^{2} \varphi=\nabla^{2} \varphi^{\prime}=0
$$

and

$\frac{\partial \varphi}{\partial x}=\frac{\partial \varphi^{\prime}}{\partial x}=0$ as $x=0$, for all $t$

$\frac{\partial \varphi}{\partial y} \rightarrow 0$ as $y \rightarrow-\infty$, for all $x, t$,

$\frac{\partial \varphi^{\prime}}{\partial y} \rightarrow 0$ as $y \rightarrow+\infty$, for all $x, t$,

Appropriate solutions which satisfy these conditions are of the form :
Cette deuxième partie comporte l'examen des oscillations stationnaires $\dot{a}$ l'interface entre deux fluides; la fréquence de l'oscillation et l'équation du profil d'onde sont déterminées au $4^{\circ}$ ordre. La míthode est celle utilisée par Penney et Price pour les oscillations en surface libre. Comme dans le cas des ondes progressives, les ampliades des harmoniques d'ordre supérieur sont réduites par la présence du fluide supérieur, c'est-à-dire que le profil de ces harmoniques devient plus sinusoüdal et que la période de l'oscillation est augmentée. L'attention est également attirée sur l'intervention d'harmoniques indéterminés dans l'équation du profil d'onde.

\section{Formulation du problème.}

Nous choisissons une origine de coordonnées 0 , située à l'interface séparant deux fluides semiinfinis, la densité du fluide supérieur étant désignée par $\rho^{\prime}$, et celle du fluide inférieur par $\rho$, l'axe Oy étant dirigé verticalement vers le haut. Le mouvement est déterminé par les potentiels de vitesse $\varphi, \varphi^{\prime}$, avec :

$$
\nabla^{2} \varphi=\nabla^{2} \varphi^{\prime}=0
$$

et

$\frac{\partial \varphi}{\partial x}=\frac{\partial \varphi^{\prime}}{\partial x}=0$ Iorsque $x=0$, pour tous les $t$

$\frac{\partial \varphi}{\partial y} \rightarrow 0$ lorsque $y \rightarrow-\infty$, pour tous les $x, t$,

$\frac{\partial \varphi^{\prime}}{\partial y} \rightarrow 0$ lorsque $y \rightarrow+\infty$, pour tous les $x, t$

I.es solutions appropriées satisfaisant à ces conditions sont de la forme :

$$
\begin{aligned}
& \varphi^{\prime}=\sum_{0}^{\infty} \alpha_{n} e^{-n y} \cos n x \\
& \varphi=\sum_{0}^{\infty} \beta_{n} e^{n y} \cos n x
\end{aligned}
$$

The interfacc may be represented by the equation :
L'interface peut être représentée par l'équation :

$$
f(x, y, t)=-y+\sum_{1}^{\infty} a_{n} \cos n x=0
$$


so that the pressure and kinematic conditions on $f=0$ are: de sorte que les conditions de pression et cinématiques pour $f=0$ sont :

$$
\begin{gathered}
p=p^{\prime} \\
\frac{\partial f}{\partial t}=\frac{\partial f}{\partial x} \frac{\partial \varphi^{\prime}}{\partial x}+\frac{\partial f}{\partial y} \frac{\partial \varphi^{\prime}}{\partial y} \\
\frac{\partial f}{\partial t}=\frac{\partial f}{\partial x} \frac{\partial \varphi}{\partial x}+\frac{\partial f}{\partial y} \frac{\partial \varphi}{\partial y}
\end{gathered}
$$

where

avec :

$$
p / \varphi=-y+\frac{\partial \varphi}{\partial t}-\frac{1}{2}\left(\varphi_{x^{2}}+\varphi_{y}^{2}\right)+\mathrm{F}(t)
$$

with a similar expression for $p^{\prime}$, all variables being measured in non-dimensional units. Substituting the expansions (2) and (3) in (4), (5) and (6), and using the notation of Penney and Price (1952), et, arec une expression analogue pour $p^{\prime}$, toutes les variables ćtant mesurées en unités sans dimensions. En faisant intervenir les développements (2) et (3) en (4), (5) et (6), et en adoptant la notation de Penney et Price (1952):

$$
e^{\lambda y} \cos \mu x=\mathrm{E}(\lambda, \mu)+\sum_{s=1}^{\infty} \cos s x[\mathrm{E}(\lambda, s-\mu)+\mathrm{E}(\lambda, s+\mu)]
$$

where

avec :

$$
\begin{aligned}
\mathrm{E}(\lambda, s) & =\mathrm{E}(\lambda,-s)=\sum_{0}^{\infty} \frac{\lambda^{n}}{2^{n} n !} \mathrm{S}_{n}(s) \\
\mathrm{S}_{n}(s) & =\sum_{-\infty}^{\infty} a_{m} \mathrm{~S}_{n-1}(s-m),
\end{aligned}
$$

and

et :

$$
\mathrm{S}_{n}(s)=\mathrm{S}_{n}(--s), \quad \mathrm{S}_{0}(0)=1, \quad \mathrm{~S}_{0}(s)=0, \quad \mathrm{~S}_{1}(s)=a_{s},
$$

the three boundary conditions become:

les trois conditions aux limites deviennent:

$$
\begin{aligned}
& \left(\rho-\rho^{\prime}\right) \sum_{1}^{\infty} a_{n} \cos n x=\rho \sum_{n=1}^{\infty} \dot{\beta}_{n}\left[\left(\mathrm{E}(n, n)+\sum_{s=1}^{\infty} \cos s x\{\mathrm{E}(n, s-n)+\mathrm{E}(n, s+n)\}\right]\right. \\
& +\rho \beta_{0}-\rho^{\prime} \dot{\alpha}_{0}-\rho^{\prime} \sum_{n=1}^{\infty} \alpha_{n}\left[\mathrm{E}(-n, n)+\sum_{s=1}^{\infty} \cos s x\{\mathrm{E}(-n, s-n)+\mathrm{E}(-n, s+n)\}\right] \\
& -\frac{1}{2} \rho \sum_{1}^{\infty} \sum_{1}^{\infty} n m \beta_{n} \beta_{m}\left[\mathrm{E}(m+n, m-n)+\sum_{1}^{\infty} \cos s x\{\mathrm{E}(m+n, s-m+n)+\mathrm{E}(m+n, s+m-n)\}\right] \\
& +\frac{1}{2} \rho^{\prime} \sum_{1}^{\infty} \sum_{1}^{\infty} n m \alpha_{n} \alpha_{m} \\
& \quad \times\left[\mathrm{E}(-m-n, m-n)+\sum_{1}^{\infty} \cos s x\{\mathrm{E}(-m-n, s-m+n)+\mathrm{E}(-m-n, s+m-n)\}\right]
\end{aligned}
$$

$$
\begin{aligned}
\sum_{1}^{\infty} \dot{a}_{n} \cos n x= & -\sum_{1}^{\infty} n \beta_{n}\left\lceil\mathrm{E}(n, n)+\sum_{1}^{\infty} \cos s x\{\mathrm{E}(n, s-n)+\mathrm{E}(n, s+n)\}\right. \\
& +\frac{1}{2} \sum_{1}^{\infty} \sum_{1}^{\infty} m n a_{m} \beta_{n}\left[\begin{array}{l}
\mathrm{E}(n, m-n)-\mathrm{E}(n, m+n)+\sum_{1}^{\infty} \cos s x\{\mathrm{E}(n, s-m+n) \\
+\mathrm{E}(n, s+m-n)-\mathrm{E}(n, s-m-n)-\mathrm{E}(n, s+m+n)\}
\end{array}\right]
\end{aligned}
$$




$$
\begin{array}{r}
\sum_{1}^{\infty} \dot{a}_{n} \cos n x=\sum_{1}^{\infty} n \alpha_{n}\left[\mathrm{E}(-n, n)+\sum_{1}^{\infty} \cos s x\{\mathrm{E}(-n, s-n)+\mathrm{E}(-n, s+n)\}\right] \\
+\frac{1}{2} \sum_{1}^{\infty} \sum_{1}^{\infty} m n a_{m} \beta_{n}\left[\begin{array}{l}
\mathrm{E}(-n, m-n)-\mathrm{E}(-n, m+n)+\sum_{1}^{\infty} \cos s x\{\mathrm{E}(-n, s-m+n) \\
+\mathrm{E}(-n, s+m-n)-\mathrm{E}(-n, s-m-n)-\mathrm{E}(-n, s+m+n)\}
\end{array}\right]
\end{array}
$$

Equating the coefficients of separate harmonics in $x$, the following relations are obtained for the $a_{n}, \alpha_{n}$, and $\beta_{n}$;
La mise en équation des coefficients de harmoniques séparés en $x$, nous donne les relations suivantes pour les $a_{n}, \alpha_{n}$, et $\beta_{n}$ :

$$
\begin{aligned}
\rho \dot{\beta}_{0}+\rho \sum_{1}^{\infty} \dot{\beta}_{n} \mathrm{E}(n, n)- & \frac{1}{2} \sum_{1}^{\infty} \sum_{1}^{\infty} \rho m n \beta_{m} \beta_{n} \mathrm{E}(m+n, m-n) \\
& -\rho^{\prime} \dot{\alpha}_{0}-\rho^{\prime} \sum_{1}^{\infty} \dot{\alpha}_{n} \mathrm{E}(-n, n)+\frac{1}{2} \sum_{1}^{\infty} \sum_{1}^{\infty} \rho^{\prime} m n \alpha_{m} \alpha_{n} \mathrm{E}(-m-n, m-n)=0 \\
\left(\rho-\rho^{\prime}\right) \alpha_{s}= & \rho \sum_{1}^{\infty} \dot{\beta}_{n}\{\mathrm{E}(n, s,-n)+\mathrm{E}(n, s+n)\}-\rho^{\prime} \sum_{i}^{\infty} \dot{\alpha}_{n}\{\mathrm{E}(-n, s-n)+\mathrm{E}(-n, s+n)\} \\
& -\frac{1}{2} \rho \sum_{1}^{\infty} \sum_{1}^{\infty} m n \beta_{m} \beta_{n}\{\mathrm{E}(m+n, s-m+n)+\mathrm{E}(m+n, s+m-n)\} \\
& +\frac{1}{2} \rho^{\prime} \sum_{1}^{\infty} \sum_{1}^{\infty} m n \alpha_{m} \alpha_{n}\{\mathrm{E}(-m-n, s-m+n)+\mathrm{E}(-m-n, s+m-n)\} \\
& \frac{1}{2} \sum_{1}^{\infty} \sum_{1}^{\infty} m n a_{m} \beta_{n}\{\mathrm{E}(n, m-n)-\mathrm{E}(n, m+n)\}-\sum_{1}^{\infty} n \beta_{n} \mathrm{E}(n, n)=0
\end{aligned}
$$

$$
\dot{a}_{s}=\frac{1}{2} \sum_{1}^{\infty} \sum_{1}^{\infty} m n a_{m} \beta_{n}\{\mathrm{E}(n, s-m+n)+\mathrm{E}(n, s+m-n)
$$$$
-\mathrm{E}(n, s-m-n)-\mathrm{E}(n, s+m+n)\}-\sum_{1}^{\infty} n \beta_{n}\{\mathrm{E}(n, s-n)+\mathrm{E}(n, s+n)\}
$$$$
\frac{1}{2} \sum_{1}^{\infty} \sum_{1}^{\infty} m n a_{m} \alpha_{n}\{\mathrm{E}(-n, m-n)-\mathrm{E}(-n, m+n)\}+\sum_{1}^{\infty} n \alpha_{n} \mathrm{E}(-n, n)=0
$$

$$
\begin{aligned}
\dot{a}_{s}=\frac{1}{2} \sum_{1}^{\infty} \sum_{1}^{\infty} m n a_{m} \alpha_{n}\{\mathrm{E}(-n, s-m+n)+\mathrm{E}(-n, s+m-n)-\mathrm{E}(-n, s-m-n) \\
-\mathrm{E}(-n, s+m+n)\}+\sum_{1}^{\infty} n \alpha_{n}\{\mathrm{E}(-n, s-n)+\mathrm{E}(-n, s+n)\}
\end{aligned}
$$

Equations (11), (13) and (15) are valid for any positive integer $s$.

\section{Solution to the fourth order.}

To this order we require the expressions :

$$
\begin{aligned}
& \left(\mathrm{E}(\lambda, 0)=1+\frac{1}{4} \lambda^{2} a_{1}{ }^{2}\right. \\
& \mathrm{E}(\lambda, 1)=\frac{1}{2} \lambda a_{1}+\frac{1}{4} \lambda^{2} a_{1} a_{2}+\frac{1}{16} \lambda^{3} a_{1}{ }^{3}
\end{aligned}
$$

Les équations (11), 13 et (15) sont valables pour n'importe quel nombre entier positif $s$.

\section{Solution au quatrième ordre.}

Pour la solution au quatrième ordre, il nous faut les expressions: 


$$
\begin{aligned}
& \mathrm{E}(\lambda, 2)=\frac{1}{2} \lambda a_{2}+\frac{1}{8} \lambda^{2} a_{1}{ }^{2} \\
& \mathrm{E}(\lambda, 3)=\frac{1}{2} \lambda a_{3}+\frac{1}{4} \lambda^{2} a_{1} a_{2}+\frac{1}{48} \lambda^{3} a_{1}{ }^{3}
\end{aligned}
$$

taken from the tables of $\mathrm{E}(\lambda, \mu)$ given by Penney and Price (page 263). Substituting these in equations (11), (13) and (15) the following differential equations are obtained, valid to the fourth order in the ratio of wave amplitude to wavelength. lesquelles sont tirées des tableaux pour $\mathrm{E}(\lambda, \mu)$ donnés par Penney et Price (page 263). En faisant intervenir ces expressions dans les équations (11), 13 et (15), nous obtenons les équaitons différentielles suivantes, valables au quatrième ordre, dans le rapport de l'amplitude à la longueur d'onde :

$$
\begin{aligned}
&\left(\rho-\rho^{\prime}\right) a_{1}=\rho \dot{\beta}_{1}\left\{1+\frac{3}{8} a_{1}^{2}+\frac{1}{2} a_{2}^{\prime}\right\}+\rho \dot{\beta}_{2} a_{1}-\rho \beta_{1}^{2} a_{1}-2 \rho \beta_{1} \beta_{2} \\
&-\rho^{\prime} \dot{\alpha}_{1}\left\{1+\frac{3}{8} a_{1}^{2}-\frac{1}{2} a_{2}\right\}+\rho^{\prime} \dot{\alpha}_{2} a_{1}-\rho^{\prime} \alpha_{1}^{2} a_{1}+2 \rho^{\prime} \alpha_{1} \alpha_{2}, \\
&\left(\rho-\rho^{\prime}\right) a_{2}=\frac{1}{2} \rho^{\prime} \dot{\beta}_{1}\left\{a_{1}+a_{1} a_{2}+a_{3}+\frac{1}{6} a_{1}^{3}\right\}+\rho \dot{\beta}_{2}\left(1+a_{1}^{2}\right)+\frac{3}{2} \rho a_{1} \dot{\beta}_{3}-\rho \beta_{1}^{2}\left(a_{2}+\frac{1}{2} a_{1}^{2}\right) \\
&-\frac{1}{2} \rho^{\prime} \dot{\alpha}_{1}\left\{-a_{1}+a_{1} a_{2}-a_{3}-\frac{1}{6} a_{1}^{3}\right)-\rho^{\prime} \dot{\alpha}_{2}\left(1+a_{1}^{2}\right)+\frac{3}{2} \rho^{\prime} a_{1} \dot{\alpha}_{3}-\rho^{\prime} \alpha_{1}^{2}\left(a_{2}-\frac{1}{2} a_{1}^{2}\right) \\
&-3 \rho \beta_{1}\left(a_{1} \beta_{2}+\beta_{3}\right)-3 \rho^{\prime} \alpha_{1}\left(a_{1} \alpha_{3}-\alpha_{3}\right),
\end{aligned}
$$

$\left.\left(\rho-\rho^{\prime}\right) a_{3}=\rho \dot{\beta}_{1}\left(\frac{1}{2} a_{2}+\frac{1}{8} a_{1}^{2}\right)+\rho \dot{\beta}_{2} a_{1}+\rho \dot{\beta}_{3}-\rho^{\prime} \dot{\alpha}_{1} \frac{1}{8} a_{1}^{2}-\frac{1}{2} a_{2}\right)+\rho^{\prime} \dot{\alpha}_{2} \alpha_{1}-\rho^{\prime} \dot{\alpha}_{3}$,

$\left(\rho-\rho^{\prime}\right) a_{4}=\rho \dot{\beta}_{1}\left(\frac{1}{2} a_{3}+\frac{1}{4} a_{1} a_{2}+\frac{1}{48} a_{1}^{3}\right)+\rho \dot{\beta}_{2}\left(a_{2}+\frac{1}{2} a_{1}^{2}\right)+\frac{3}{2} \rho \dot{\beta}_{3} a_{1}+\rho \dot{\beta}_{4}$

$$
-\rho^{\prime} \dot{\alpha}_{1}\left(-\frac{1}{2} a_{3}+\frac{1}{4} a_{1} a_{2}-\frac{1}{48} a_{1}^{3}\right)-\rho^{\prime} \dot{\alpha}_{2}\left(-a_{2}+\frac{1}{2} a_{1}^{2}\right)+\frac{3}{2} \rho^{\prime} \dot{\alpha}_{3} a_{1}-\rho^{\prime} \dot{\alpha}_{4}
$$

$$
\left.\begin{array}{l}
\dot{\alpha}_{1}=-\beta_{1}\left(1+\frac{1}{8} a_{1}^{2}-\frac{1}{2} a_{2}\right)-\beta_{2} a_{1}, \\
\dot{\alpha}_{2}=-\beta_{1}\left(a_{1}+\frac{1}{12} a_{1}^{3}-a_{3}\right)-2 \beta_{2}\left(1+a_{1}^{2}\right)-3 \beta_{3} a_{1}, \\
\dot{\alpha}_{3}=-\beta_{1}\left(\frac{3}{2} a_{2}+\frac{3}{8} a_{1}^{2}\right)-3 \beta_{2} a_{1}-3 \beta_{3}, \\
\dot{\alpha}_{4}=-\beta_{1}\left(2 a_{3}+a_{1} a_{2}+\frac{1}{12} a_{1}^{3}\right)-2 \beta_{2}\left(a_{1}^{2}+2 a_{2}\right)-6 \beta_{3} a_{1}-4 \beta_{4} \\
\dot{a}_{1}=\alpha_{1}\left(1+\frac{1}{8} a_{1}^{2}+\frac{1}{2} a_{2}\right)-\alpha_{2} a_{1}, \\
\dot{\alpha}_{2}=\alpha_{1}\left(-a_{1}-\frac{1}{12} a_{1}^{3}+a_{3}\right)+2 \alpha_{2}\left(1+a_{1}^{2}\right)-3 \alpha_{3} a_{1}, \\
\dot{\alpha}_{3}=\alpha_{1}\left(-\frac{3}{2} a_{2}+\frac{3}{8} a_{1}^{2}\right)-3 \alpha_{2} a_{1}+3 \alpha_{3}, \\
\dot{a}_{4}=\alpha_{1}\left(-2 a_{3}+a_{1} a_{2}-\frac{1}{12} a_{1}^{3}\right)+2 \alpha_{2}\left(a_{1}^{2}-2 \alpha_{2}\right)-6 \alpha_{3} a_{1}+4 \alpha_{4}
\end{array}\right\}
$$

These equations may be solved iteratively in the usual way making use of the fact that $a_{n}, \alpha_{n}, \beta_{n}$ are all at least of order $\left(a_{1}\right)^{n}$. This is
Ces équations peuvent être résolues par la méthode classique des différences finies (itération), en tirant parti du fait que $a_{n}, \alpha_{n}, \beta_{n}$, sont tous au 
readily established in the same way as for the free surface oscillations. The first four coeficients in the profile equation (3) are as follows : moins de l'ordre $\left(a_{1}\right)^{n}$, ce que l'on établira aisément de la même manière que pour les oscillations en surface libre. Les quatre premiers coefficients de l'équation du profil (3) sont les suivants :

$$
\begin{aligned}
& a_{1}=\mathrm{A} \sin \sigma t+\mathrm{A}^{3} \frac{\left(3 \rho^{2}-6 \rho \rho^{\prime}-\rho^{\prime 2}\right)}{32\left(\rho+\rho^{\prime}\right)^{2}} \sin \sigma t+\mathrm{A}^{3} \frac{\left(\rho^{2}-\rho \rho^{\prime}+\rho^{\prime 2}\right)}{16\left(\rho+\rho^{\prime}\right)^{2}} \sin 3 \sigma t \\
& a_{2}=\mathrm{J}+\mathrm{K} \cos 2 \sigma t+\mathrm{L} \cos 4 \sigma t, \\
& a_{3}=\mathrm{A}^{3} \frac{\left(3 \rho^{\circ}-10-10 \rho^{\prime}+3 \rho^{\prime 2}\right)}{32\left(\rho+\rho^{\prime}\right)^{2}}(3 \sin \sigma t-\sin 3 \sigma t), \\
& a_{4}=\mathrm{M}+\mathrm{N} \cos 2 \sigma t+\mathrm{P} \cos 4 \sigma t,
\end{aligned}
$$

where

avec :

$$
\begin{aligned}
& \mathrm{J}=\frac{\mathrm{A}^{2}}{4}\left(\frac{\rho-\rho^{\prime}}{\rho+\rho^{\prime}}\right)+\rho \mathrm{A}^{4} \frac{\left(2 \rho^{2}-\rho \rho^{\prime}-2 \rho^{\prime 2}\right)}{32\left(\rho+\rho^{\prime}\right)^{3}} \\
& \mathrm{~K}:=-\frac{\mathrm{A}^{2}}{4}\left(\frac{\rho-\rho^{\prime}}{\rho+\rho^{\prime}}\right)+\mathrm{A}^{4} \frac{\left(25 \rho^{3}-13 \rho^{\prime 3}-59 \rho^{2} q^{\prime}+41 \rho \rho^{\prime 2}\right)}{192\left(\rho+\rho^{\prime}\right)^{3}} \\
& \mathrm{~L}=-\cdots \mathrm{A}^{4} \frac{\left\{67\left(\rho^{3}-\rho^{3}\right)-179 \rho \rho^{\prime}\left(\rho-\rho^{\prime}\right)\right\}}{1344\left(\rho+\rho^{\prime}\right)^{3}} \\
& \mathrm{M}=\mathrm{A}^{4} \frac{\left(\rho-\rho^{\prime}\right)\left(\varphi^{2}-6 \rho^{\prime}+\rho^{\prime 2}\right)}{8\left(\rho+\rho^{\prime}\right)^{3}}
\end{aligned}
$$

and

et :

$$
\mathrm{P}=\mathrm{A}^{4} \frac{\left(\rho-\rho^{\prime}\right)\left(\rho^{2}-6 \rho o^{\prime}+\rho^{\prime 2}\right)}{24\left(\rho+\rho^{\prime 3}\right)}
$$

The coefficient $\mathrm{N}$ is indeterminate as can be seen from the form of equations (16) (17) and (18) which give :
On voit, d'après la forme des équations (16), (17) et (18), que le coefficient $\mathrm{N}$ est indéterminé. Ces équations nous domnent :

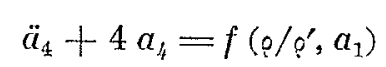

the complementary function giving an arbitrary term in $\cos 2, t$. It is clear that the indeterminate terms in the equation to the wave profile are:

$$
\begin{gathered}
\mathrm{A} \cos x \sin \sigma t, \mathrm{~A}^{4} \cos 4 x \cos 2 \sigma t, \\
\mathrm{~A}^{0} \cos 9 x \sin 3 \sigma t,
\end{gathered}
$$

and these are not specified by the condition of strict periodicity nor the inilial wave profile. To this extent the problem of stationary oscillations of finite amplitude, unlike the problem of progressive waves, does not possess a unique soIution.

The frequency of oscillation is found from the relation : dans laquelle la fonction complémentaire fournit un terme arbitraire en cos 2 ot. Les termes indéterminés dans l'équation du profil d'onde sont manifestement :

$$
A \cos x \sin \sigma t, \quad \mathrm{~A}^{4} \cos 4 x \cos 2 \sigma t,
$$

$$
A^{\prime} \cos 9 x \sin 3 \sigma t
$$

lesquals ne sont spécifiés, ni par la condition de périodicité rigoureuse, ni par le profil d'onde initial. Dans cette mesure, et contrairement au problème des ondes progressives, le problème des oscillations stationnaires d'amplitude finie ne possède aucune solution unique.

La fréquence d'oscillation s'obtient par la relation :

$$
\sigma^{2}=\left(\frac{\rho-\rho^{\prime}}{\rho+\rho^{\prime}}\right)\left\{1-\frac{A^{2}}{4} \frac{\rho^{2}+\rho^{\prime 2}}{\left(\rho+\rho^{\prime}\right)^{2}}\right\}
$$


It is readily seen that these results reduce to those of Penney and Price as $\varphi^{\prime} / \varphi \rightarrow 0$. For $\rho^{\prime} / \rho \neq 0$, the period of oscillation is increased, while the wave profile is appreciably more sinusoidal throughout the major portion of the wave period.
Il est évident que ces résultats se réduisent à ceux de Penney et Price lorsque $\varphi^{\prime} / p \rightarrow 0$. Pour $\varphi^{\prime} / \varphi \neq 0$, la période de l'oscillation augmente, et le profil d'onde reste nettement plus sinusoïdal pendant la plus grande partie de la période de l'onde.

\title{
Bibliographie
}

Penney, W. G. and Price, A.T. (1952). - Finite periodic stationary gravity waves in a perfect fluid. Phil. Trans. Roy. Soc., (A) 244, 254-284.

\section{NOTRE FRONTISPICE}

\author{
(Cf page 450)
}

MORLAND (Sir Samuel, baromet) (1625-1695).

Cet hydraulicien, né vers 1625 à Sulhamstead (Berkshire), conmença par etre diplomate, sous Cromwell, puis « maitre des mécaniques " du roi Charles II.

Il se donna pour tāche d'élever les eatxx de la Tamise jusqu'à In plus haute corniche du château de Windsor, et ensuite jusqu'à 80 pieds au-dessus; les ouvrages imaginés dans ce but se révélèrent plus dispendieux que véritablement utiles. Mais cela fut pour leur auteur l'occasion d'écrire un traité de "l'Elévation des eaux par toute sorte de machines, réduite à la mesure, au poids et à la balance $\$$ (1683) et de faire paraitre en 1685, à Paris, un mémoire sur " l'Elévation des eaux par le moyen d'un nouveau piston et corps de pompe et $d^{\prime} u n$ nouveau mouvement cyclo-elliptique, en rejetant l'usage de toute sorte de manivelles ordinaires \%; il est regrettable d'avoir à constater que cet opuscule, qui était sans dorte une étape dans la theorie des pompes rotatives, renferne quelques niaiseries et ne soit pas exempt d'une pointe de charlatanisme.

En 1697, vraisemblablement après son décès, dont la date est incertaine, parut sa meilleure cuvre: "Hydrostatique, ou instructions concernant les travaux hydrauliques $)$, dont une importante partie, consacrée aux puits et pompes, était destinée a rendre accessible au public les notions relatives à ces ouvrages et à ces machines.

Mais sir Samuel Morland se signala par sa collaboration, en France, à l'invention du porte-voix, et surtout par la grande et justifiée réputation qu'il s'acquit comme constructeur, en Angleterre, d'appareils de physique et notamment de baromètres.
Sir Samuel MORLAND, Bt. (1625-1695)

Born at Sulhamstead in Berkshire in about 1625, Sir Samuel Morland was a hydranlician zuho began his carecr as a diplomat under Cromevell and then became the "Master of the Royal Machines" under King Charles II.

He set himself the tash of pumping water from the Thames up to the topmost cernice of Windsor castle, and then to a height 80 feet above it. Although the devices he designed for the purpose turned out to be rather more costly than effective, they nevertheless provided him with an opportinity for writing a wo the ling of water by machines of all kinds, reduced to principles of measurement, weight, and the balance" (1683), and for publishing an article th Pans in 1685 on "the lifting of water by means of a new piston and pump body having a cyclo. cliptic molion and rendering all the nstal crank mechanisms superintedy. It is a great. pity that this work, which was theorbedly a step for oard in the development of rotary punt theory, also contans a minber of downright vacuous statements, point of sheer quackery.

Morland's best zeorl, on "Hydrostatics, or instructions concenting hydranlic works" zas published in 1697 , probably after his death, the date of which is uncertain. Wells and pumps are discussed at length in this treatise. mainly aith the purpose of acquainting the gencral public with the principal ideas underlying their design.

Morland is also knozen for his collaboration with French scien tists in inventing the megaphone, and cspecially for the well. deserved reputation he buth ut in England as a designer of physical instruments, among which barometers in particular. 